\title{
Intergenerational Public Goods: Transnational Considerations
}

\author{
by \\ Todd Sandler* \\ School of Economic, Political and Policy Sciences \\ University of Texas at Dallas, GR 31 \\ 800 W. Campbell Road \\ Richardson, TX 75080-3021 \\ $1-972-883-6725$ \\ Fax 1-972-883-6486 \\ tsandler@utdallas.edu
}

November 2008

\begin{abstract}
This paper analyzes public goods whose benefits cross countries' borders and generations. Once provided, transnational intergenerational public goods (TIPGs) are assets that generate benefits for subsequent generations. For treaties involving TIPGs, a coalition of rich developed countries are apt to shoulder more of the provision burden then a coalition of poor developing countries. This burden inequality worsens if the developed countries unilaterally display altruism to future generations; nevertheless, altruistic benefits may offset reduced spillovers. When current generations strategize with respect to future generations, provision and efficiency suffer; however, the hypothesized unequal burden sharing improves somewhat. For alternative aggregator technologies of public supply, these one-sided burdens may be partly ameliorated.
\end{abstract}

Keywords: Environmental Treaties; Externalities; Intergenerational Efficiency; Leader-Follower Behavior; Noncooperative Games; Public Goods

JEL codes: H87, Q58

*Vibhooti Shukla Professor of Economics and Political Economy, University of Texas at Dallas. The author is grateful to two anonymous reviewers for their helpful comments on an earlier version of the paper. 


\section{INTRODUCTION}

In recent years, there has been an increased interest in transnational public goods (TPGs), whose nonrival and nonexcludable benefits transcend national borders (see, e.g., Ferroni and Mody, 2002; Kaul et al., 1999; Kindleberger, 1986; Sandler, 1997, 1998, 2004; Secretariat of the International Task Force on Global Public Goods, 2006). This interest has been sparked by concerns for the environment, security, health, and governance. In particular, the analysis of TPGs offers insights into treaty formation and adherence (Barrett, 2003; Sandler and Sargent, 1995), and is particularly relevant for the study of pollution, the commons, and other issues associated with resource and public economics. Many of these TPGs possess benefits that transgress not only national borders but also generations as actions yesterday or today impact the consumption of tomorrow's generations (John and Pecchenino, 1997; John et al., 1995). Curtailing stratospheric ozone depletion or limiting the accumulation of greenhouse gases (GHGs) through treaty or other collective action mechanism generates benefits into the distant future. This is also true of preserving natural habitats (e.g., barrier reefs or forests), mapping the earth or the heavens, accumulating knowledge, preserving culture, and eradicating pests or diseases. Past investigations have either studied the transnational (see, e.g., Ferroni and Mody, 2002) or the transgenerational dimension (see, e.g., Myles, 1997; Rangel, 2003, 2005; Sandler, 1982; Sandler and Smith, 1976). Only a few past studies investigated the interplay of transnational and transgenerational benefits associated with intergenerational public goods (IPGs) (see, e.g., John and Pecchenino, 1997; Sandler, 1978). A related issue is the intergenerational responsibility that a present generation has in regards to addressing the harms caused by past generations. ${ }^{1}$ Although this ethical concern is important, this paper solely examines efficiency aspects.

The primary purpose of the current study is to extend the analysis of transnational IPGs 
(TIPGs) to analyze the implications for treaty making and allocative efficiency when there are two coalitions of countries that differ in terms of economic development and, of course, national income. This scenario is characteristic of treaties such as the Montreal Protocol on ozone shield depleters or the Kyoto Protocol on global warming. My theoretical investigation shows that treaties involving TIPGs will place a much larger provision burden on the more developed countries even in the absence of distributional considerations. As such, TIPGs create an exploitation hypothesis founded, in part, on temporal considerations where the more farsighted and altruistic countries carry a disproportionately large burden for the more myopic countries. ${ }^{2}$ In this paper, disproportionality refers to one coalition covering more of the provision expenditure of the TPIG. A secondary purpose is to permit the present generation to act strategically vis-à-vis the future generation; this strategizing ameliorates somewhat the unequal burden placed on developed countries with respect to TIPGs. A third purpose is to introduce alternative aggregation technologies of public supply (i.e., how individual provisions add to the overall consumption level) that play a crucial role in how generations can trade backward and forward in time to support TIPGs. This role has gone unrecognized and can influence intergenerational burden sharing and efficiency.

The analysis indicates that efforts to account for intergenerational benefits - say, through taxation or debt accumulation - place greater TIPG burdens on the more farsighted coalition. Actions to account for spatial externalities may not improve welfare from an intergenerational standpoint. For intergenerational Pareto optimality, treaties concerning TIPGs must adjust for spatial and temporal spillovers; most participants are unlikely to display such altruism to their neighbors, their neighbor's children, or their children's children. Sophisticated efforts by a current generation to adjust for the anticipated TIPG provision of a subsequent generation reduce efficiency and redistribute TIPG provision burdens from rich to poor countries. Some TIPG 
aggregator technologies are more conducive than others to intergenerational recontracting and to tying together generations. Since a diverse set of aggregators are associated with TIPGs, some TIPGs actually possess more favorable provision prognosis in the sense of a Nash equilibrium that is nearer to the Pareto optimum.

The remainder of the paper contains five sections. In Section II, the baseline model is presented. Section III displays the consequences of alternative methods for a coalition to internalize the associated intergenerational externalities, embodied in the TIPG. In Section IV, the current generation anticipates the allocation decisions of the next generation. In Section V, the impact of alternative aggregator technologies on the provision of TIPGs is presented. Concluding remarks follow in Section VI.

\section{BASELINE NASH MODEL}

This model contains two periods, $j=1,2$, with two generations, $i=1,2$. The first generation lives for two periods, while the second generation lives for just one period - i.e., period 2 - until the model's endpoint. Any number of overlapping generations can be accommodated. In addition, the model allows for two coalitions of countries - developed $(D)$ and less-developed $(L)$ - where each coalition acts as a unitary player. The analysis here ignores the issue of

coalition formation and stability which is dealt with elsewhere. ${ }^{3}$ Coalition $D$ possesses a higher aggregate national income than coalition $L$ and identical single-period utility functions to those of $L$. Each generation in coalition $D$ and $L$ is assumed to possess a single individual; additional individuals would necessitate another index of summation, without providing any further insights. The TIPG is denoted by $Q$ for the aggregate consumption levels and by $q$ for individual contribution levels, whereas the private numéraire good is given by $y$. The utility or welfare of each participant is indicated by $u$, while income is denoted by $I$. For most variables, there are 
two superscripts and one subscript. Thus, $q_{D}^{i j}$ represents coalition $D$ 's $i^{\text {th }}$ generation's

contribution to the TIPG during period $j$, while $u_{D}^{i j}$ is coalition $D$ 's $i^{\text {th }}$ generation's welfare during period $j$. To satisfy sufficiency conditions for a maximum, each utility function is assumed to be strictly quasi-concave and increasing in both the private and public goods. Moreover, both goods are normal with positive income elasticities. These assumptions are sufficient for uniqueness and existence of a Nash equilibrium, with Nash reaction paths displaying the shapes later given in the figures (Cornes and Sandler, 1996; Cornes et al., 1999).

At the outset, the first generation behaves naïvely by taking the TIPG provision of the next generation as given. Later, a more sophisticated leadership role for the first generation is assumed. The naïve representation fosters intuition and allows complications to be added in a natural progression. The Nash concepts are needed to evaluate the implications of the leaderfollower intergenerational model.

When the first generation in $D$ acts independently, the generation maximizes its multiperiod utility function, which is the sum of the single-period utilities over the generation's two-period lifetime, subject to its two-period linear budget constraint. ${ }^{4}$ To specify the objective function, the aggregator technology for the TIPG must be indicated. This aggregator is initially summation, so that $Q^{1}=q_{D}^{11}+q_{L}^{11}$ in period 1 and $Q^{2}=q_{D}^{11}+q_{D}^{12}+q_{D}^{22}+q_{L}^{11}+q_{L}^{12}+q_{L}^{22}$ in period 2 . The second expression follows because consumption increases with contributions made in any period by either generation in the two coalitions. This representation allows for benefit spillovers across coalitions and time, so that a TIPG provided in the first period augments the two generations' consumption in both coalitions over the two periods. Thus, the TIPG is an intergenerational asset. I have not permitted depreciation of the TIPG in the second period; this could be easily accommodated by weighting amounts of the public good provided in period 1 by 
discount factor $0<\delta<1$ (see Myles, 1997). The potential influence of depreciation will, however, be acknowledged in a later footnote.

Coalition D's first generation chooses its provision of $y$ and $q$ in the two periods to maximize

$$
u_{D}^{11}\left(y_{D}^{11}, Q^{11}\right)+u_{D}^{12}\left(y_{D}^{12}, Q^{12}\right)+\lambda\left[I_{D}^{11}+I_{D}^{12}-y_{D}^{11}-p q_{D}^{11}-y_{D}^{12}-p q_{D}^{12}\right]
$$

while taking TIPG provision by $D$ 's second generation and the provision by both generations in coalition $L$ as given. In equation (1), $\lambda$ is an unspecified Lagrangian multiplier, while $p$ is the unit price of the TIPG. The unit price of the private good is normalized to equal one. The budget constraint in brackets merely equates income in the two periods to lifetime expenditures. The resulting first-order conditions (FOCs) can be expressed as:

$$
\begin{aligned}
& M R S_{Q y D}^{11}+M R S_{Q y D}^{12}=p, \\
& M R S_{Q y D}^{12}<p,
\end{aligned}
$$

where $M R S_{Q y D}$ is coalition D's marginal rate of substitution (MRS) between the TIPG and the private good. The superscripts on the MRSs indicate the generation and time period, respectively. Equation (2) indicates that generation 1 chooses a positive TIPG provision in period 1 so as to equate the public good's marginal benefits over its two-period time horizon to the public good's relative price. In contrast, inequality (3) is consistent with $q_{D}^{12}=0$. Since $q_{D}^{11}$ benefits coalition $D$ for two periods at a unit cost of $p$, while $q_{D}^{12}$ benefits this coalition for only a single period at the same cost, there is no gain from waiting a period to provide the TIPG. ${ }^{5}$ Because coalition $D$ 's second generation only lives for period 2, its constrained objective is:

$$
u_{D}^{22}\left(y_{D}^{22}, q_{D}^{11}+q_{D}^{22}+q_{L}^{11}+q_{L}^{22}\right)+\lambda\left[I_{D}^{22}-y_{D}^{22}-p q_{D}^{22}\right]
$$


where $q_{D}^{12}$ and $q_{L}^{12}$ have been set to zero. Generation 2's choice of $y_{D}^{22}$ and $q_{D}^{22}$ gives the following FOC:

$$
M R S_{Q y D}^{22} \leq p,
$$

in which an equality is associated with $q_{D}^{22}>0$ and a strict inequality is associated with $q_{D}^{22}=0$. The Nash FOCs, given in equations (2)-(3) and (5), do not adjust for the benefit spillovers conferred on the other coalition or generation. An identical set of FOCs hold in coalition $L$.

\section{Intergenerational Pareto optimality}

The notion of optimality employed here is that of intergenerational Pareto optimality, which corresponds to a position from which it is not possible to improve the welfare of any individual without harming some other individual in either some generation or coalition (Myles, 1997; Rangel, 2003; Sandler, 1982; Sandler and Smith, 1976). The associated FOCs can be derived in two alternative fashions: (i) maximizing the sum of the two generations' multiperiod utility function in coalition $D$ and $L$, subject to an appropriate income constraint; or (ii) maximizing one individual's (generation's) welfare in a given period, subject to the constancy of $u_{r}^{i j}$ in other periods, generations, and coalitions, and subject to the income constraints. ${ }^{6}$ The relevant FOCs are:

$$
\begin{aligned}
\sum_{r=D, L}\left(\sum_{j=1}^{2} M R S_{Q y r}^{1 j}+M R S_{Q y r}^{22}\right)=p & \text { for the choice of } q_{D}^{11} \text { and } q_{L}^{11}, \\
\sum_{r=D, L} M R S_{Q y r}^{22} \leq p & \text { for the choice of } q_{D}^{22} \text { and } q_{L}^{22} .
\end{aligned}
$$

A comparison of the intergenerational Pareto optimality conditions in equations (6)-(7) and those of the Nash equilibrium in equation (2) and (5) reveals some interesting insights. In equation (2), the benefits conferred on $L$ 's first generation - i.e., $\left(M R S_{Q y L}^{11}+M R S_{Q y L}^{12}\right)$ - are left 
out. Moreover, the intergenerational external benefits conferred on coalition $D$ 's and $L$ 's second generation are ignored in equation (2), so that $\left(M R S_{Q y D}^{22}+M R S_{Q y L}^{22}\right)$ is absent. Thus, externalities are over time and space; the presence of more generations and/or coalitions would simply mean that more of these spillover benefits are ignored. By leaving out spatial and temporal benefits, the Nash FOCs are associated with an undersupply of the TIPG relative to the criterion of intergenerational Pareto optimality, thus justifying international treaties that bolster the supply of such goods. In the past, the analysis of treaties focused on providing TPGs where the temporal dimension was ignored (Barrett, 2003; Sandler and Sargent, 1995).

The analysis thus far implies that a treaty that only internalizes the spatial externalities will not achieve intergenerational Pareto optimality because temporal externalities must also be taken into account. Consider nuclear energy where the near-term externalities are positive, while the longer-term intergenerational externalities are negative owing to storage risks of highly radioactive daughter elements. In this scenario, sharing nuclear energy technologies internalizes positive spatial externalities, while worsening negative intergenerational externalities if storage technologies are not addressed. Another instance is the transnational provision of a drug (e.g., antiretroviral drugs in the treatment of HIV/AIDS), whose increased use limits its effectiveness over time. Thus, correcting one set of externalities may not, on net, make things better off from an intergenerational Pareto efficiency perspective. Treaties between developed and lessdeveloped countries coalitions may have disappointing results owing to differing capacities for the coalitions to internalize the intergenerational portion of the externalities.

\section{Graphical treatment}

A graphical treatment facilitates an investigation of further implications in Sections III and IV. 
This treatment draws on constrained iso-utility curves first introduced by Cornes and Sandler (1984) that are now a standard device in the study of public goods. For graphical purposes, I initially assume that $q_{D}$ and $q_{L}$ are only provided in period 1 , so that superscript indices on the $q$ s are not needed for the time being to denote the generation or the period of provision. In the intergenerational Pareto optimality conditions [see equation (6)], there will still be six MRS terms with three $M R S$ expressions associated with each of the two coalitions.

[Figure 1]

In Figure 1, $q_{L}$ and $q_{D}$ are displayed on the vertical and horizontal axis, respectively. The U-shaped II contour represents one income-constrained iso-utility curve for coalition $D$, where the curve's slope equals

$$
d q_{L} / d q_{D}=-1+\left[p /\left(M R S_{Q y D}^{11}+M R S_{Q y D}^{12}\right)\right] .
$$

For each level of $q_{L}$, the Nash FOC in equation (2) is satisfied at the iso-utility curve's minimum point, where the slope is zero. Iso-utility curves to the northwest, such as $I^{\prime} I^{\prime}$, represent a higher level of utility than $I I$, since, for a given level of $q_{D}$ provision, coalition $D$ receives more spillovers from $L$. The horizontal lines $x$ and $y$ depict two alternative levels of coalition $L$ 's TIPG provision. Coalition $D$ 's Nash reaction path, $N^{D}$, is downward sloping and steeper than a line with slope -1 owing to the income normality assumption (Cornes and Sandler, 1996). ${ }^{7}$

[Figure 2]

In Figure 2, the graphical analysis incorporates the iso-utility curves (e.g., $I^{L} I^{L}$ ) and the Nash reaction path, $N^{L}$, for coalition $L$. The latter's iso-utility contours are C-shaped because $L$ 's graphs are $90^{\circ}$ translations of those of coalition $D$ owing to the labeling of the axes. Coalition $L$ 's Nash reaction path connects the point on each of $L$ 's iso-utility curves where the slope is infinite. In contrast to $N^{D}$, path $N^{L}$ is flatter than a line with slope -1 . The Nash equilibrium 
occurs at $N$ at the unique point where the two reaction paths intersect. By drawing a ray with slope -1 from $N$ to the $x$ axis, one can locate the total provision, $Q^{N}=q_{D}+q_{L}$, associated with the Nash equilibrium. In Figure 2, the shaded region between $I^{L} I^{L}$ and $I^{D} I^{D}$ indicates Paretosuperior allocations, insofar as the iso-utility curves through these points represent higher welfare levels for both coalitions. The associated $Q$ levels - if a ray with slope -1 is drawn to the horizontal axis - exceed that of $Q^{N}$, thus establishing the undersupply of the TIPG.

\section{PITFALLS OF INTERNALIZING TRANSGENERATIONAL EXTERNALITIES}

In this section, we show that when coalitions of developed and less-developed countries meet to negotiate a treaty on providing a TIPG, key asymmetries are apt to result in outcomes to the disadvantage of coalition $D$. Efforts by coalition $D$ to overcome myopia may be burdensome.

\section{Intergenerational altruism}

Intergenerational altruism by coalition $D$ involves a social welfare function, whereby a positive weight, $\mu \leq 1$, is put on the second generation's welfare. This results in an FOC for the first generation as follows:

$$
M R S_{Q y D}^{11}+M R S_{Q y D}^{12}+\mu M R S_{Q y D}^{22}=p
$$

which implies a greater provision of the TIPG for each $q_{L}$ level. With this unilateral intergenerational altruism, the slope of $D$ 's iso-utility curves becomes:

$$
d q_{L} / d q_{D}=-1+\left[p /\left(M R S_{Q y D}^{11}+M R S_{Q y D}^{12}+\mu M R S_{Q y D}^{22}\right)\right]
$$

Compared with equation (8), the denominator in the bracketed expression is larger, so that the iso-utility curves' minimum points are displaced rightward owing to altruism. This means that each level of $q_{L}$ is now associated with a larger provision of $q_{D}$. In Figure 3, this results in a 
rightward shift in $N^{D}$ to $N_{G}^{D}$. The new Nash equilibrium at $N_{2}$ indicates a redistribution in TIPG burdens from coalition $L$ to coalition $D$. In the process, coalition $L$ 's welfare improves since its iso-utility curve (not shown) through $N_{2}$ is necessarily at a higher level than the curve through $N_{1}$ as $L$ 's free-riding opportunity increases. Even though $D$ 's iso-utility curve through $N_{2}$ is lower than that through $N_{1}, D$ 's welfare is not necessarily reduced because its social welfare function has changed owing to altruism. That is, the iso-utility curves used to construct $N^{D}$ are noncomparable to those used to derive $N_{G}^{D}$. The overall level of TIPG provision has increased to $Q_{G}^{N}$, with coalition $D$ carrying more of the provision burden as $q_{D}$ rises and $q_{L}$ declines.

[Figure 3]

If this theme is pursued further so that coalition $D$ also accounts for spatial or transnational spillovers, then $N^{D}$ shifts further right to $N_{G T}^{D}$ and the overall TIPG supply increases to $Q_{G T}^{N}$, with $D$ taking on still more of the burden. In the extreme, $N^{D}$ may shift sufficiently rightward to give a corner solution on the horizontal axis, where coalition $L$ is afforded a free ride. ${ }^{8}$ Coalition $D$ may regret its spatial and generational awareness (generosity) if the burden becomes too lopsided. Unilateral internalization of spatial or generational spillovers will not achieve intergenerational Pareto efficiency - the iso-utility curves through $N_{3}$ (not displayed) form a lens-shaped region, as in Figure 2, where both coalitions will be better off than at $N_{3}$. Externalities have not been fully internalized owing to $L$ 's lack of altruism.

Next suppose that both coalitions are intergenerationally altruistic but have different life expectancies, with coalition $D$ 's population having a longer life span than $L$ 's population. This agrees with reality. Thus, there is more generational overlap in $D$ which should result in a higher 
altruistic weight being applied to the second generation's derived benefits from the TIPG. This means that the shift out of $D$ 's Nash reaction path is fuller than that of coalition $L$, so that $D$ 's relative burden increases even though it gains from $L$ 's altruism. Since coalition $L$ 's altruism is understandably small because of its more limited income, coalition $D$ must expect to take on more TIPG burdens. This translates into lopsided treaty terms for TIPGs.

\section{A subsidy scheme}

I now consider a way that allows a coalition to better account for its subsequent generation's interests through a self-imposed tax/subsidy scheme. Coalition $D$ employs the subsidy scheme on its own actions in order to rectify the myopia that it anticipates from member nations, whose current public good provision collectively ignores benefits generated for the subsequent generation. Unilateral intergenerational awareness in this multi-coalition scenario refers to a situation where one coalition alone accounts for the benefits that its public good provision in the first period confers on the coalition's second generation. To achieve such awareness, coalition $D$ may institute a subsidy that can achieve the FOC in equation (9). Thus, suppose that $D$ institutes a subsidy of $t$ per unit of $q_{D}$, financed by a lump-sum tax of $T$ to sterilize any income effects in coalition $D$. If the TIPG is only provided in the first period, consistent with Figure 3, then the first generation in $D$ maximizes

$$
u_{D}^{11}\left(y_{D}^{11}, q_{D}+q_{L}\right)+u_{D}^{12}\left(y_{D}^{12}, q_{D}+q_{L}\right),
$$

subject to

$$
I_{D}^{11}+I_{D}^{12}+T=y_{D}^{11}+y_{D}^{12}+(p-t) q_{D},
$$

where account is taken of the subsidy and the lump-sum tax. Upon simplification, the following FOC results: 


$$
M R S_{Q y D}^{11}+M R S_{Q y D}^{12}=(p-t)
$$

If the subsidy equals $\mu M R S_{Q y D}^{22}$, then equation (9) is satisfied from the viewpoint of coalition $D$.

Although coalition D's unilaterally imposed tax/subsidy affords coalition $L$ a freer ride, D's altruism may justify its augmented provision despite fewer spillovers. Thus, past characterizations of unilateral actions as being necessarily self-defeating (Hoel, 1991; Sandler, 1996) do not capture altruistic-motivated taxes or subsidies in the presence of a TIPG.

Since developed countries have a more advanced public finance system and the wealth to be altruistic (i.e., altruism is income elastic), the scenario depicted here is likely. In the case of curbing GHGs and ozone depleters, developed countries have instituted such subsidy and tax schemes. For ozone depleters, $N^{L}$ is sufficiently flat not to result in much reduced spillovers for $D$, since less-developed countries used relatively small amounts of ozone-shield-depleting substances. This is not the case of GHGs, where the drive to development will mean large increases in GHGs in developing countries, such as India and China. These large increases over time will eventually hurt the developed countries - a motivation for US failure to sign the Kyoto Protocol. Clearly, both the Montreal Protocol and the Kyoto Protocol place most of the burden of the associated TIPGs on the developed countries. The Montreal Protocol allowed lessdeveloped ("Article 5") countries a ten-year reprieve from chlorofluorocarbons (CFCs) cutbacks (Benedick, 1991). In fact, these less-developed countries were actually allowed to increase their CFC use up to a threshold over ten years, after which a Multilateral Fund (supported by the developed countries) would subsidize these countries' switchover to more ozone-benign substances. The Kyoto Protocol exempted most less-developed countries from any GHG reductions (Sandler, 2004). For the Oslo Protocol on sulfur emissions, percentage cutbacks were much more lenient to the less-developed European countries, such as Portugal, Greece, and 
Poland, that are more dependent on coal-fired power plants for their energy (Finus and Tjøtta, 2003). Thus, the model's burden prediction is accurate in three instances.

\section{SOPHISTICATED DECISION MAKING}

I now extend the analysis to allow the first generation to anticipate the actions of the subsequent generation, when deciding its contributions to the TIPG. In particular, the first generation no longer takes the second generation's provision of the TIPG as given; instead, the first generation realizes that the second generation in $D$ and $L$ will be responding to what the first generation supplied in period 1 . Thus, the first generation acts as the leader and the second generation acts as the follower, which is a natural progression given their temporal sequencing. The first generation accounts for the second generation's negatively sloped reaction paths: $q_{D}^{22}=q_{D}^{22}\left(Q^{1}\right)$ and $q_{L}^{22}=q_{L}^{22}\left(Q^{1}\right)$. That is, the first generation in $D$ chooses $y_{D}^{11}, q_{D}^{11}, y_{D}^{12}$, and $q_{D}^{12}$ to maximize

$$
\begin{aligned}
u_{D}^{11}\left(y_{D}^{11}, Q^{1}\right) & +u_{D}^{12}\left[y_{D}^{12}, q_{D}^{11}+q_{D}^{12}+q_{D}^{22}\left(Q^{1}\right)+q_{L}^{11}+q_{L}^{12}+q_{L}^{22}\left(Q^{1}\right)\right] \\
& +\lambda\left[I_{D}^{11}+I_{D}^{12}-y_{D}^{11}-p q_{D}^{11}-y_{D}^{12}-p q_{D}^{12}\right] .
\end{aligned}
$$

As before, we ignore discounting and depreciation. The associated FOCs are:

$$
\begin{aligned}
& \operatorname{MRS}_{Q y D}^{11}+\operatorname{MRS}_{Q y D}^{12}\left[1+\frac{\partial Q^{2}}{\partial q_{D}^{22}} \frac{d q_{D}^{22}}{d Q^{1}}+\frac{\partial Q^{2}}{\partial q_{L}^{22}} \frac{d q_{L}^{22}}{d Q^{1}}\right]=p \\
& M R S_{Q y D}^{12}<p
\end{aligned}
$$

In equation (12), the multiplicative $\partial Q^{1} / \partial q_{D}^{11}$ in the last two terms in the square brackets is suppressed, because it equals one. The last two multiplicative expressions on the left-hand side of equation (12) are negative owing to the negative slopes of the second generation's reaction paths - i.e., $d q_{D}^{22} / d Q^{1}<0$ and $d q_{L}^{22} / d Q^{1}<0$. 
When condition (12) is compared with equation (2), we discover that the first generation in $D$ reduces its provision of the TIPG as a means to induce the second generations in $D$ and $L$ to expand their TIPG provision. Thus, the sophisticated choice of $q_{D}^{11}$ is smaller than that of the naïve Nash model presented earlier. The implied leader-follower behavior between the two generations results in the first generation shifting more the TIPG burden onto the second generation. Sophisticated behavior on the part of the first generation means that the resulting equilibrium will depart even further than the Nash equilibrium from the intergenerational Pareto efficiency condition in equation (6). Thus, opportunistic behavior by the first generation has adverse consequences for efficiency.

In equation (13), the first generation only invests in the TIPG during the first period. If depreciation is positive, then a nonstrict inequality would apply with some positive provision $\left(q_{D}^{12}>0\right)$ possible in the second period. The second generation in $D$ maximizes the expression in equation (4), because, as the follower, it still abides by Nash behavior with a FOC corresponding to equation (5). Consequently, the second generation still does not account for spatial spillovers. Coalition $L$ 's first and second generations satisfy FOCs analogous to equations (12), (13), and (5).

If altruism characterizes the first generation in $D$, the following FOC applies:

$$
M R S_{Q y D}^{11}+M R S_{Q y D}^{12}\left(1+\frac{\partial Q^{2}}{\partial q_{D}^{22}} \frac{d q_{D}^{22}}{d Q^{1}}+\frac{\partial Q^{2}}{\partial q_{L}^{22}} \frac{d q_{L}^{22}}{d Q^{1}}\right)+\mu M R S_{Q y D}^{22}\left(1+\frac{\partial Q^{2}}{\partial q_{D}^{22}} \frac{d q_{D}^{22}}{d Q^{1}}+\frac{\partial Q^{2}}{\partial q_{L}^{22}} \frac{d q_{L}^{22}}{d Q^{1}}\right)=p
$$

Strategizing by the first generation reduces both the second and third marginal benefit expressions on the left-hand side, compared to equation (9). A subsidy based on the third term is now less effective owing to adverse efficiency consequences of burden shifting by the first generation as it takes advantage of its leadership position. 


\section{Graphical display}

The graphical device, introduced earlier, can now be modified to allow for the provision of the TIPG in both generations, with the first generation acting a leader. In Figure $4, q_{D}^{11}$ and $q_{D}^{22}$ are displayed on the horizontal and vertical axes, respectively. $N^{1}$ is the Nash reaction path for $D$ 's first generation, while $N^{2}$ is the Nash reaction path for $D$ 's second generation. As leader, the first generation in $D$ seeks the tangency between its iso-utility curve, $I I$, and the reaction path of $D$ 's second generation. The total quantity, $Q_{D}=q_{D}^{11}+q_{D}^{22}$, shrinks from $0 R$ to $0 F$ in Figure 4 when the Nash amount, $O R$, is compared with the leader-follower amount, $O F$, assuming that $q_{L}^{11}$ and $q_{L}^{22}$ are temporarily fixed in coalition $L$. As generation 1 shifts the burden onto generation 2, the former is better off and the latter is worse off at $S$ compared with the Nash equilibrium. This follows because generation 1's iso-utility curve through $S$ is higher than that through $N$. Generation 2's isoutility curve (not shown) through $S$ is a lower level of utility, since it is leftward of the isoutility curve (also not shown) through $N$. An analogous two-generation diagram applies for coalition $L$. In Figure 4, the extent of intergenerational burden shifting depends on the slope of $N^{2}$-i.e., the shift is greater when $N^{2}$ is steeper. If $N^{2}$ is the dashed horizontal line, then there is no shift since $S$ becomes $N$ - leadership makes no difference to provision.

\section{[Figure 4]}

In Figure 5, the Nash reaction path, $N^{D}$, for coalition $D$ 's two generations is displayed with $Q_{D}$ on the horizontal axis and $Q_{L}\left(=q_{L}^{11}+q_{L}^{22}\right)$ on the vertical axis. The shape of this path follows from Figure 4, where each increase in $Q_{L}$ would cause reaction paths $N^{1}$ and $N^{2}$ to 
shift to the left and down. Consequently, $N$ would move to the left and down, implying a reduced provision of $Q_{D}$ for each increase in $Q_{L}$. As a result, $D$ 's Nash reaction curve relating $Q_{D}$ to alternative levels of $Q_{L}$ is negatively sloped in Figure 5. Similarly, coalition $L$ 's reaction path is also negatively sloped. The relative slopes of the two coalitions' Nash reaction paths again follow from income normality. $N^{D}$ is drawn further out from the vertical axis, compared with $N^{L}$ 's position relative to the horizontal axis owing to coalition $D$ 's higher income. Because TIPGs are typically income normal goods, $D$ is anticipated to display a greater preference than $L$ for the TIPG for each level of spillovers. Thus, the Nash equilibrium, $N$, indicates a relatively greater provision burden for coalition $D$. In a treaty involving a TIPG, this implies that $D$ will supply relatively more than coalition $L$ owing to income differences

[Figure 5]

In the case of sophisticated provision by the first generation in both coalitions, the impact the implied leader-follower behavior must be viewed in this two-generation, two-coalition context. I return to Figure 4 to derive the slope of $D$ 's leader-follower reaction path, $L F^{D}$, in Figure 5. Each increase in $Q_{L}$ will displace point $S$ in Figure 4 in the direction of the arrow, so that $Q_{D}$ and $Q_{L}$ are inversely related. This, in turn, means that $D$ 's leader-follower reaction path is downward sloping as displayed. Moreover, this path is to the left of $N^{D}$ because, for every $Q_{L}, Q_{D}$ is smaller for $S$ compared with $N$. By analogous reasoning, $L F^{L}$ must also be below $N^{L}$. A fuller shift will characterize coalition $D$ 's leader-follower path since there is a greater difference between $Q_{D}$ associated with Nash than with leader-follower behavior, owing to larger provision levels in $D$ than those in $L$.

When $N$ is compared with $S$ in Figure 5, coalition $D$ is able to shed more of its burden 
than coalition $L$, because generation 1's efforts to burden generation 2 does more to reduce TIPG provision in the coalition that provides more of the public good. Nevertheless, coalition $D$ still carries relatively more of the TIPG burden. Sophisticated behavior limits burden inequality among coalitions, but at the expense of intergenerational Pareto efficiency as the overall provision level decreases - i.e., provision at $S$ is less than that at $N$.

Next suppose that only the first generation in coalition $D$ acts strategically, so that the equilibrium is where $L F^{D}$ intersects $N^{L}$. Consequently, coalition $D$ is able to shed more TIPG burden. Even so, coalition $D$ assumes much of the TIPG burden and so will agree to treaties that relieve coalition $L$ of much of its TIPG responsibility. Hence, the one-sided conditions of the Montreal Protocol, the Kyoto Protocol, and other recent treaties also follows from a more sophisticated strategic analysis. Ethical norms - i.e., those responsible for the past accumulation of pollutants should assume the greater cleansing burden - are not needed to explain recent treaties' provision.

With altruism, coalition $D$ assumes even larger burdens. This follows because unilateral altruism on the part of $D$ will shift its $L F^{D}$ path to the right (not shown), thereby displacing the equilibrium in the southeast direction. In virtually all scenarios, coalition $D$ provides free-riding opportunities to coalition $L$ regarding the provision of TIPGs.

\section{AGGREGATOR TECHNOLOGIES AND TIPGs}

Hirshleifer (1983) first introduced the aggregation technology of public supply (henceforth, aggregator technology), which indicates how individual contributions combine to determine the overall level of the public good, available for consumption by benefit recipients. Prior to Hirshleifer's concept, the default aggregator was summation in which public good contributions were merely summed to ascertain the overall amount of the good. Two aggregator technologies 
have proven especially important in applications. For a weakest-link public good, the smallest contribution level fixes the amount of the public good. When trying to stem the spread of a pest or to contain nuclear waste, the country exercising the least vigilance determines the extent of the infestation or containment, respectively. In contrast, the largest contribution fixes the overall level of a best-shot public good. For example, the research team expending the greatest effort is best poised to make the breakthrough that then benefits the current and future generations. Finding a cure is a best-shot intergenerational public good, where efforts are redundant once the cure is uncovered.

A weakest-link aggregator is associated with matching behavior insofar as contributions below the smallest provision level utilize resources with no payoff. Moreover, free riding has no payoffs since the minimum contribution is then zero and no one benefits. A best-shot aggregator results in a single contributor privileging everyone to the benefits. These aggregators may have better efficiency implications than summation. For example, a weakest-link public good may result in a near-efficient provision level when contributors have similar tastes and income as a representative agent's provision is matched by other providers (Mueller, 2003).

With best-shot TIPGs, a future generation can improve or upgrade the inherited amount of the TIPG. Because future generations tend to be better off than preceding generations, TIPG improvements are anticipated over time. For example, cures for some diseases and/or improved control for some pests may come many generations hence as knowledge is accumulated. Bestshot public goods allow generations not to be saddled with an unacceptable level of a TIPG. Thus, I focus my remarks about recontracting among generations on weakest-link TIPGs.

Weakest-link TIPGs can come in myriad forms: some that inhibit recontracting among generations and others that permit recontracting. In the former, an early generation's limited provision restricts the level of the TIPG, and hence the well-being, for all future generations in 
both coalitions. Consider the following weakest-link technology:

$$
Q^{1}=\min \left\{q_{D}^{11}, q_{L}^{11}\right\}
$$

and

$$
Q^{2}=\min \left\{q_{D}^{11}, q_{L}^{11}, q_{D}^{22}, q_{L}^{22}\right\}
$$

where a small $q^{11}$ can tie the hands of the next generation, since a larger $q^{22}$ would have no influence. Irreversible changes to an environment caused by an economic activity can fit this scenario, where $q$ denotes efforts to, say, cut emissions or actions to keep from surpassing an ecosystem's carrying capacity.

This scenario means that the current generation must use asset taxes and debt to achieve the ability to provide a higher level of $q^{11}$ in the interest of the subsequent generation(s). Arrangements to borrow from future generations are essential so that they are not impoverished by their predecessors' underprovision of a TIPG. The problem is that such unilateral actions by the first generation in coalition $D$ will not achieve much because a small level of $q_{L}^{11}$ (owing to its limited income) is not rectified. Coalition $D$ must also ensure that $q_{L}^{11}$ matches the "acceptable" level of $q_{D}^{11}$. This requires a treaty where coalition $D$ uses its borrowing capacity to subsidize $q_{D}^{11}$ and $q_{L}^{11}$, thus leading to an especially lopsided burden being placed on coalition $D$ now and into the future. Thus, unequal burden sharing has an intergenerational component.

Given this aggregator technology, the exploitation potential is extreme, because it is reinforced by coalition and intergenerational capacity considerations. Because the current generation is likely poorer and more limited in know-how than subsequent generations, the former is particularly disadvantaged, but pivotal in the allocation of the TIPG. Without any efforts to account for the other coalition or generations, $D$ 's first generation satisfies 


$$
M R S_{Q y D}^{11}+M R S_{Q y D}^{12}=p \text { for } q_{D}^{11} \leq q_{L}^{11},
$$

which differs greatly from the intergenerational Pareto efficiency condition,

$$
\sum_{r=D, L}\left(\sum_{j=1}^{2} M R S_{Q y r}^{1 j}+M R S_{Q y D}^{22}\right)=4 p .
$$

The right-hand side of equation (18) includes $4 p$, since $q_{r}^{i i}, i=1,2$ and $r=D, L$, must be brought up to the same level.

Introducing leader-follower considerations between the two generations is more conducive to achieving intergenerational Pareto efficiency, because the first generation in $D$ or $L$ anticipates that the next generation matches the first generation's provision - i.e., reaction paths are positively sloped. Consequently, this anticipated positive response results in additional MRS expressions (not shown) on the left-hand side of equation (17) (see e.g., Cornes and Sandler, 1996, Chapter 10). This, in turn, means that the leader-follower reaction path would lie above the Nash reaction path, thereby augmenting provision compared with naïve behavior by the first generation. Leader-follower behavior places even greater burdens on coalition $D$ than Nash behavior as it must bring coalition $L$ 's provision levels up to a higher standard.

A more hopeful situation arises when the weakest-link aggregator can permit intergenerational recontracting, so that inadequate or limited provision by a preceding generation does not constrain a future generation. The aggregator technology is now:

$$
Q^{1}=\min \left\{q_{D}^{11}, q_{L}^{11}\right\}
$$

and

$$
Q^{2}=\max \left[\min \left\{q_{D}^{11}, q_{L}^{11}\right\}, \min \left\{q_{D}^{12}, q_{D}^{22}, q_{L}^{12}, q_{L}^{22}\right\}\right] .
$$

Equation (20) indicates that a subsequent or past generation can reverse an earlier period's shortfall. For disease eradication, insufficient efforts in an earlier period can be corrected in a 
subsequent period, when the vaccination program manages to surpass herd immunity as was true in the late 1970s for smallpox. Earlier generations' inability to eradicate polio may soon be reversed through efforts coordinated by the World Health Organization (WHO) and funded by developed countries. Since subsequent generations are wealthier than preceding generations, the problem will eventually be addressed. Unequal burden sharing is more limited than the last weakest-link case because the current generation in $D$ does not have to make up for the capacity of future generations in $L$.

Other related aggregators - e.g., weaker link - would permit less stringent recontracting opportunities even for representations more akin to equations (15)-(16), since complete matching is not required. The introduction of aggregator technologies to the study of TIPGs offers a new perspective on intergeneration allocative efficiency.

\section{CONCLUDING REMARKS}

This paper investigates TIPGs whose benefits transcend borders and generations. With a standard summation aggregator, TIPGs imply that developed countries will shoulder a much larger burden as they make up for shortfalls associated with less-developed countries and myopia. This lopsided burden sharing arises owing to income and altruism differences between developed and less-developed countries. Altruism toward future generations are likely to increase as a coalition of countries becomes wealthy. Countries that can barely meet their own subsistence do not have the luxury to generously support TIPGs that benefit current and future generations. Although TIPG burdens will be carried by developed countries, this does not mean that they are necessarily worse off because rich countries gain from their actions to improve the well-being of their next generation. Unilateral altruism, while burdensome and supportive of free riding, may be in rich countries' interests. More sophisticated decision making by the first 
generations in coalition $D$ and $L$ shifts some of the TIPG burden back on coalition $L$, but this shift is apt to be modest. The analysis indicates that treaties to provide TIPGs will be particularly one-sided, with less-developed countries taking on little of the provision responsibility - a prediction consistent with the stipulations of recent environmental treaties. When the analysis is extended to include alternative aggregator technologies of public supply, a richer set of burden-sharing scenarios arise. For weakest-link TIPGs, coalition $D$ 's burdens are less one-sided if the weakest-link aggregator permits recontracting by future generations, where decisions today can be changed tomorrow, as in the case of disease eradication. In this scenario, the current generation in $D$ only has to bring the provision of the TIPG up to an acceptable level today, leaving tomorrow's generation to improve provision further. The amendment process of treaties permits recontracting between generations as supply parameters of TIPGs change over time. Even modest treaties that provide for amendments can ultimately promote TIPG provision by putting an adjustment process in place, which is precisely what the Vienna Convention and the Long-Range Transboundary Air Pollution Convention did for controlling chlorofluorocarbons and sulfur, respectively. 


\section{Footnotes}

1. From an ethical perspective, the current and future generations in developed countries may be viewed as responsible for assuming greater clean-up burdens than developing countries for, say, global warming, insofar as developed countries' subsequent generations gained from the pollution of their ancestors (Gosseries, 2004).

2. Mancur Olson (1965) formulated the exploitation hypothesis of collective action, where the large (rich) agents shoulder a disproportionate burden of the public good for the small (poor) agents. In particular, Olson indicated that the rich nations spend a greater percentage of their national income on the public good than their poorer counterpart.

3. Coalition stability requires internal and external stability, with the former requiring that no coalition country gains from leaving and the latter requiring that no noncoalition country gains from joining (de Zeeuw, 2008; Rubio and Ulph, 2007; Ulph, 2004). In a recent paper for identical countries, de Zeeuw (2008) showed that large stable coalitions, such as assumed here, can form, given "static farsighted stability" where countries' deviation from cooperation is inhibited by a trigger mechanism (e.g., tit-for-tat). Earlier contributions demonstrated that coalitions can be large when reaction functions are steep and Stackelberg behavior, as assumed here, applies (Barrett, 2003). I ignore the coalition formation and stability issue to focus instead on intergenerational gaming in a two-coalition world, where endowments differ greatly between the coalitions. This is an unexplored issue.

4. For simplicity, a linear budget constraint is assumed with a constant relative price for the TIPG. A multiperiod transformation function could, instead, be introduced, which allows the private and public goods to be produced in each period at marginal cost. For the TIPG, this marginal cost may rise over time. In the first-order conditions below, the relevant period's marginal rate of transformation ratio would then replace $p$. Although this change will potentially 
affect the allocation of TIPG between periods, it will not alter the qualitative results concerning inefficiency or burden sharing. Thus, the price (and therefore marginal cost) is assumed constant for the two period.

5. Provision in period 2 may be justified if depreciation is at a sufficient rate so that the FOCs become:

$$
\begin{aligned}
& M R S_{Q y D}^{11}+\delta M R S_{Q y D}^{12}=p, \\
& M R S_{Q y D}^{12} \leq p .
\end{aligned}
$$

An equality in $\left(3^{\prime}\right)$ implies that $q_{D}^{12}>0$. By reducing the remaining amount of the TIPG over time, depreciation induces the first generation to supply less of the TIPG in the first period and a positive amount in the second period. This added complication does not change the paper's qualitative findings and is, thus, ignored.

6. Typically, this intergenerational optimality concept ignores discounting future generations' utility, so that all generations' gains from the IPG are treated equally (Sandler and Smith, 1976). However, Myles (1997) used discounting in his analysis.

7. Obviously, $N^{D}$ does not have to be linear.

8. Other things constant, the greater the difference in income between the two coalitions, the more likely will be the presence of a corner solution. 


\section{References}

Barrett, S. A. (2003). Environment and Statecraft: The Strategy of Environmental TreatyMaking. New York: Oxford University Press.

Benedick, R. E. (1991). Ozone Diplomacy. Cambridge, MA: Harvard University.

Cornes, R. and Sandler, T. (1984). Easy riders, joint production, and public goods. Economic Journal, 94, pp. 580-98.

Cornes, R. and Sandler, T. (1996). The Theory of Externalities, Public Goods, and Club Goods, $2^{\text {nd }}$ ed. Cambridge, U.K.: Cambridge University Press.

Cornes, R., Hartley, R. and Sandler, T. (1999). Equilibrium existence and uniqueness in public good models: an elementary proof via contraction. Journal of Public Economic Theory, 1, pp. 499-509.

de Zeeuw, A. (2008). Dynamic effects on the stability of international environmental agreements. Journal of Environment Economics and Management, 55, pp. 163-74.

Ferroni, M. and Mody, A. eds. (2002). International Public Goods: Incentives, Measurement, and Financing. Boston: Kluwer Academic Publishers.

Finus, M. and Tjøtta, S. (2003). The Oslo Protocol and sulfur reduction: the great leap forward? Journal of Public Economics, 87, pp. 2031-48.

Gosseries, A. 2004. Historical emissions and free riding. Ethical Perspective, 11, pp. 36-60.

Hirshleifer, J. (1983). From weakest-link to best-shot: the voluntary provision of public goods. Public Choice, 41, pp. 371-86.

Hoel, M. (1991). Global environmental problems: the effects of unilateral actions taken by one country. Journal of Environmental Economics and Management, 20, pp. 55-70.

John, A. A. and Pecchenino, R. A. (1997). International and intergenerational environmental externalities. Scandinavian Journal of Economics, 99, pp. 371-87. 
John, A. A., Pecchenino, R. A., Schimmelpfennig, D. E. and Schreft, S. L. (1995). Short-lived agents and the long-lived environment. Journal of Public Economics, 58, pp. 127-41.

Kaul, I., Grunberg, I. and Stern, M. A. eds. (1999). Global Public Goods: International Cooperation in the $21^{\text {st }}$ Century. New York: Oxford University Press.

Kindleberger, C. P. (1986). International public goods without international government. American Economic Review, 76, pp. 1-13.

Mueller, D. C. (2003). Public Choice III. Cambridge, U.K.: Cambridge University Press.

Myles, G. (1997). Depreciation and intergenerational altruism in the private provision of public goods. European Journal of Political Economy, 13, pp. 725-38.

Olson, M. (1965). The Logic of Collective Action. Cambridge, MA: Harvard University Press.

Rangel, A. (2003). Forward and backward intergenerational goods: why is social security good for the environment? American Economic Review, 93, pp. 813-34.

Rangel, A. (2005). How to protect future generations using tax-base restrictions. American Economic Review, 95, pp. 314-46.

Rubio, S. J. and Ulph, A. (2007). An infinite-horizon model of dynamic membership of international environmental agreements. Journal of Environmental Economics and Management, 54, pp. 296-310.

Sandler, T. (1978). Interregional and intergenerational spillover awareness. Scottish Journal of Political Economy, 25 pp. 273-84.

Sandler, T. (1982). A theory of intergenerational clubs. Economic Inquiry, 20, pp. 191-208.

Sandler, T. (1996). A game-theoretic analysis of carbon emissions. In The Political Economy of Environmental Protection: Analysis and Evidence. Roger Congleton (eds), Ann Arbor: University of Michigan Press, pp. 251-72.

Sandler, T. (1997). Global Challenges: An Approach to Environmental, Political, and Economic 
Problems. Cambridge, U.K.: Cambridge University Press.

Sandler, T. (1998). Global and regional public goods: a prognosis for collective action. Fiscal Studies, 19, pp. 221-47.

Sandler, T. (2004). Global Collective Action. Cambridge, U.K.: Cambridge University Press.

Sandler, T. and Sargent, K. (1995). Management of transnational commons: coordination, publicness and treaty formation. Land Economics, 71, pp. 145-62.

Sandler, T. and Smith, V. K. (1976). Intertemporal and intergenerational pareto efficiency. Journal of Environmental Economics and Management, 2, pp. 151-59.

Secretariat of the International Task Force on Global Public Goods. (2006). Meeting Global Challenges: International Cooperation in the National Interest: Cross-Cutting Issues. Stockholm: Erlanders Infologistics Väst AB.

Ulph, A. (2004). Stable international environmental agreements with a stock pollutant, uncertainty and learning. Journal of Risk and Uncertainty, 29, pp. 53-74. 


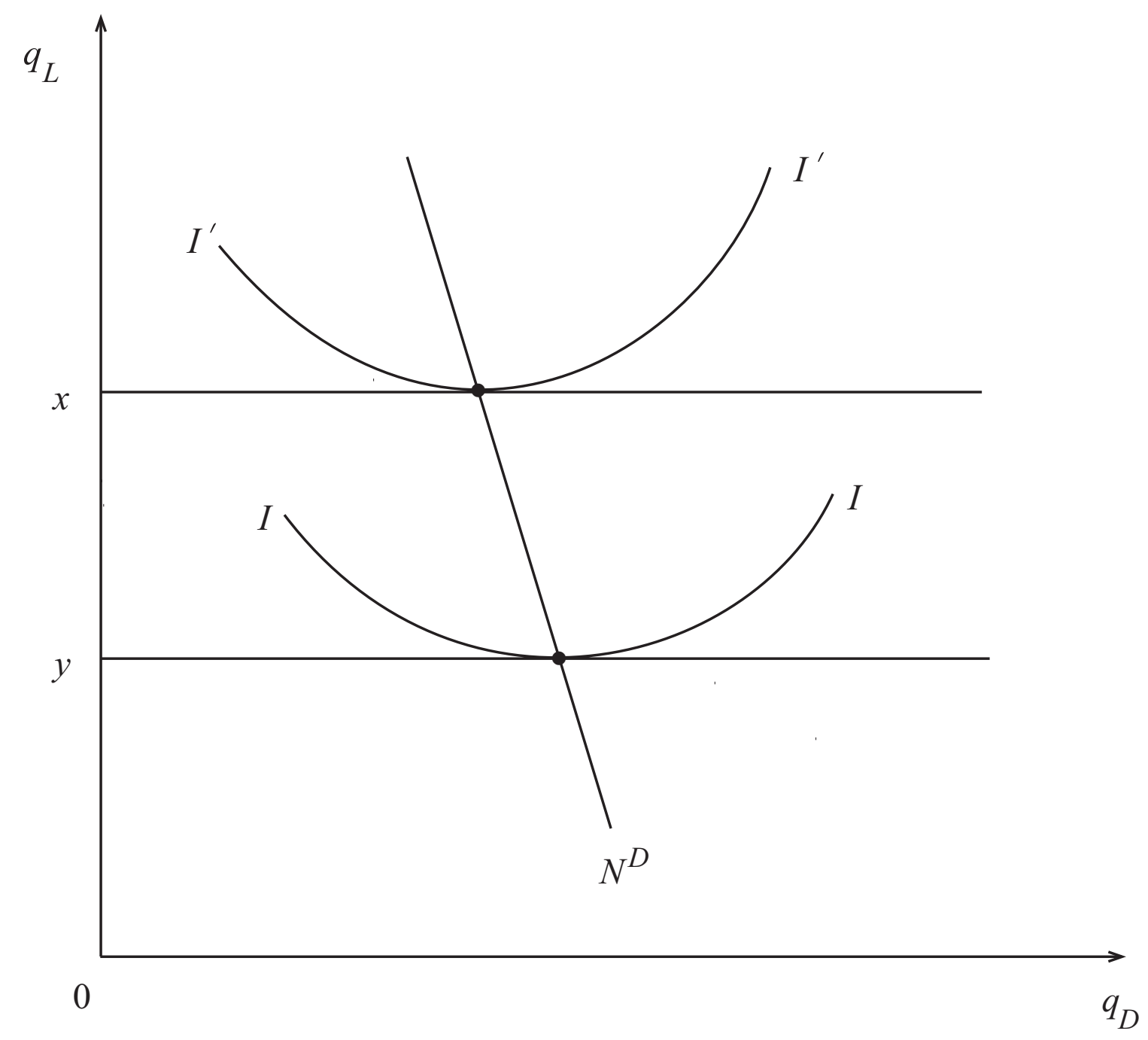

Figure 1. Nash reaction paths for $D$ coalition 


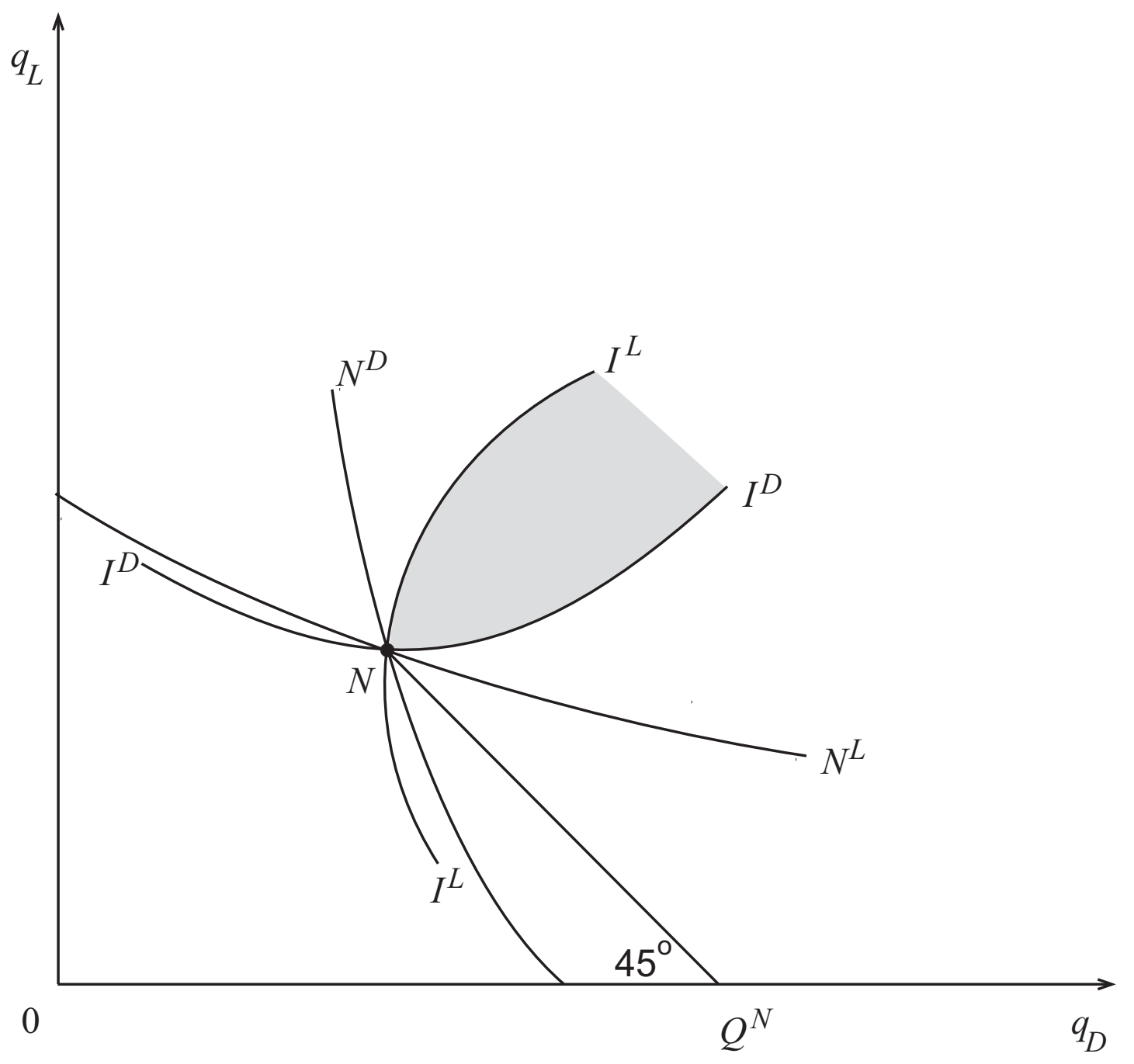

Figure 2. Nash equilibrium and Nash reaction paths 


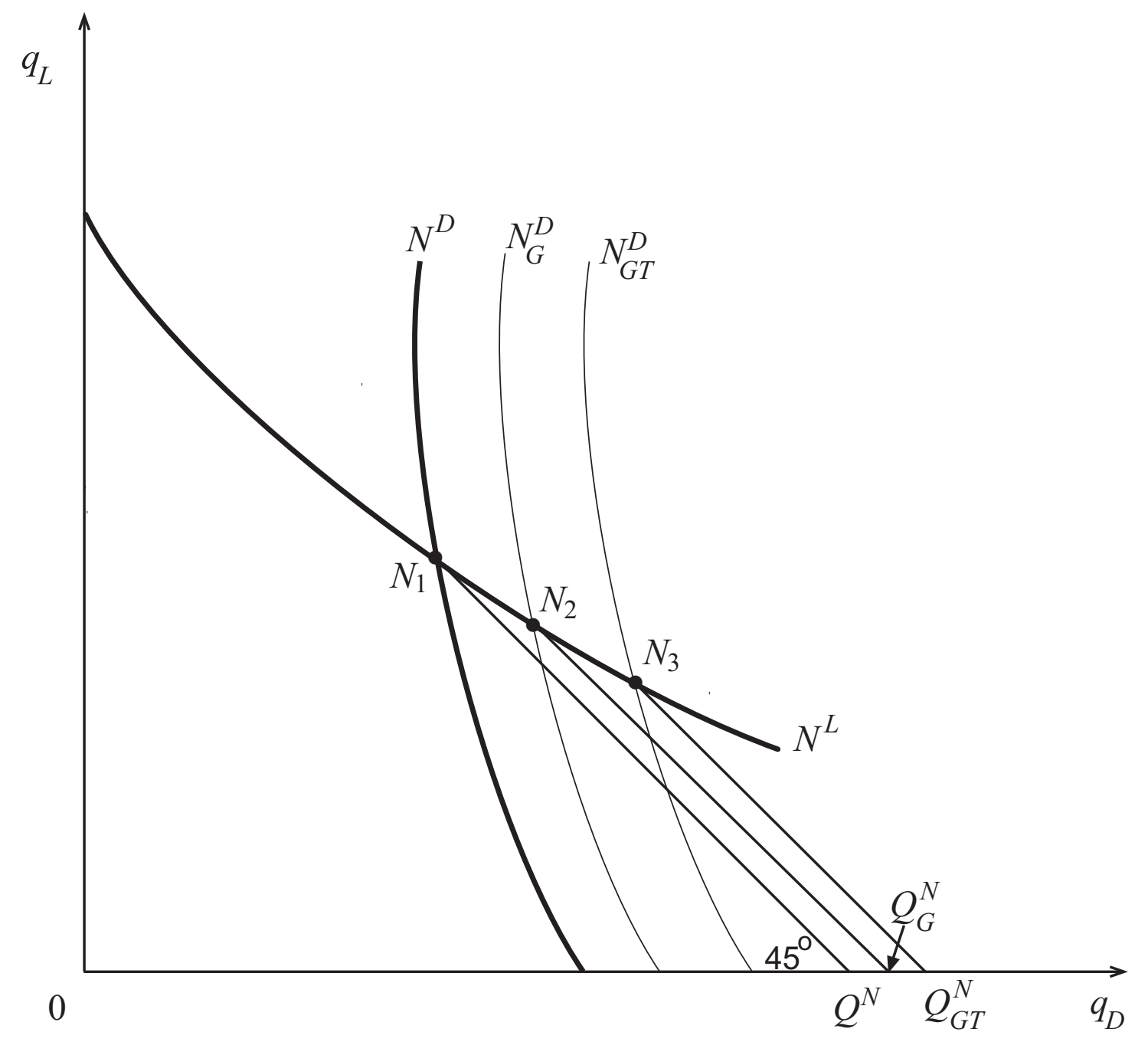

Figure 3. Unilateral increased intergenerational responsibility 


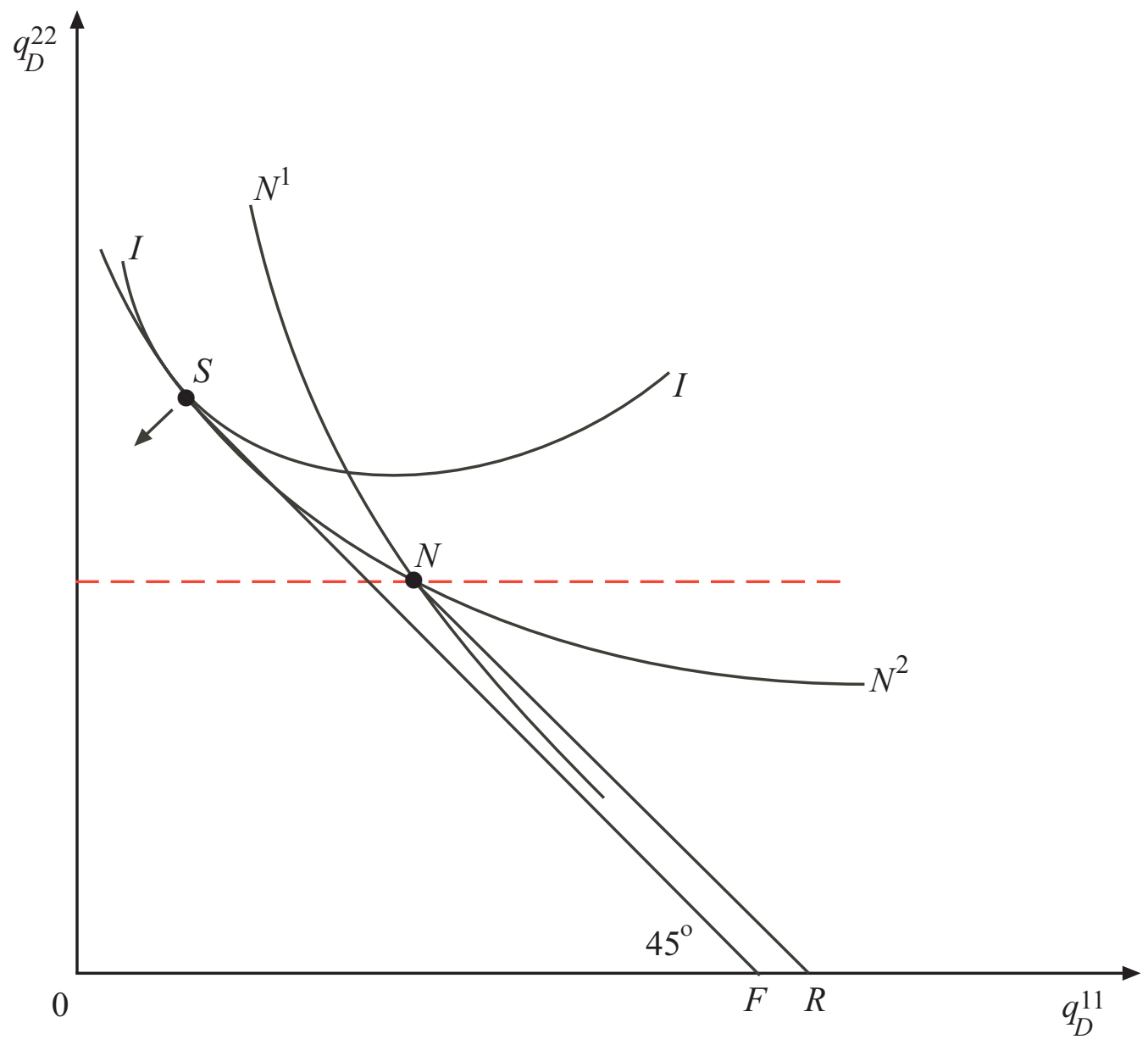

Figure 4. Intergenerational leader-follower behavior in coalition $D$ 


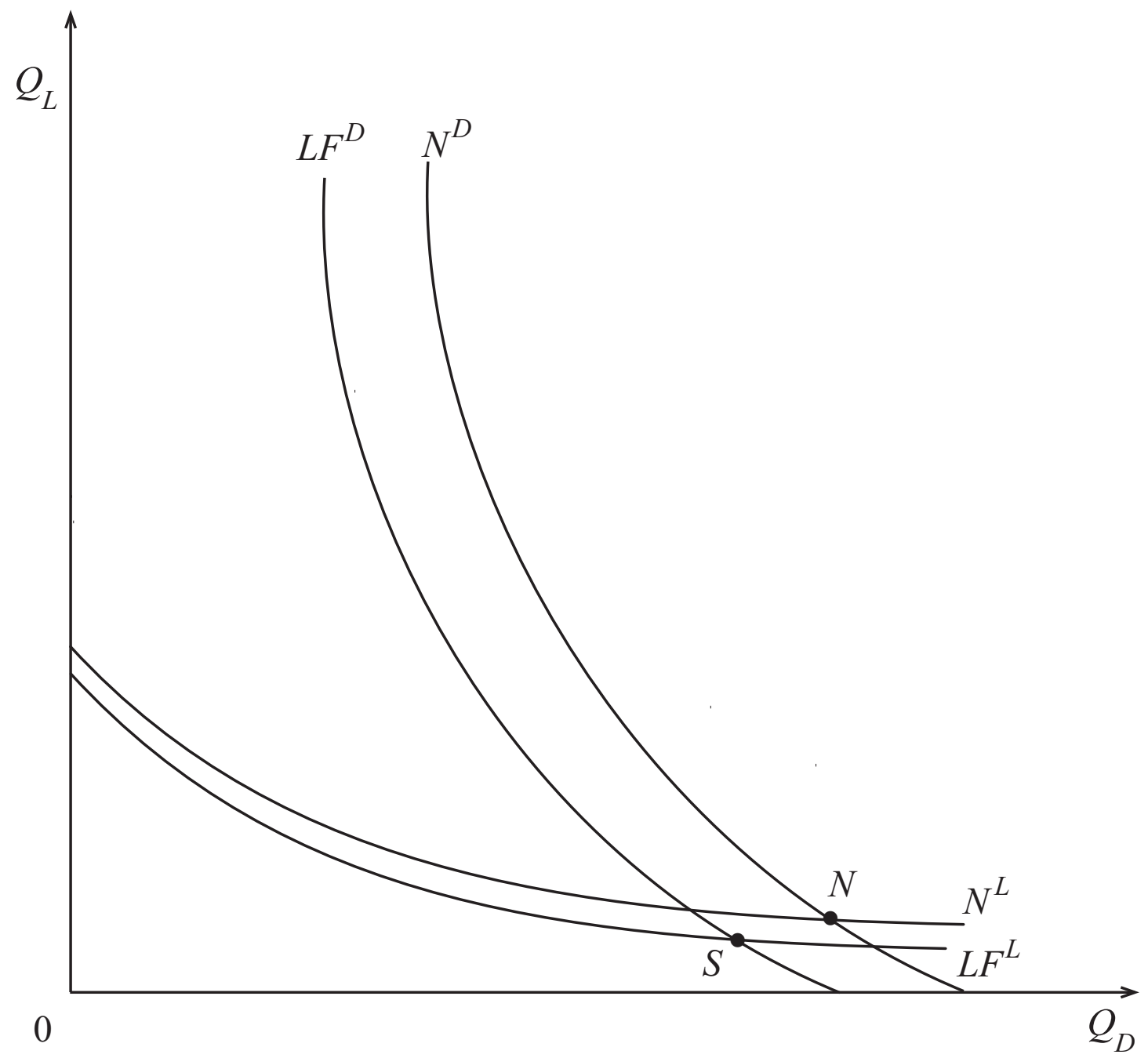

Figure 5. Sophisticated intergenerational equilibrium 